lower BMI (difference between children of smoking vs non smoking fathers, $0.8 \mathrm{~kg} / \mathrm{m}^{2}, 95 \%$ CI 0.1 to 1.4 ) and children of mothers reporting vigorous physical activity had lower BMI, cholesterol, ApoB/ApoA1 levels and decreased odds for overweight/obesity. Independent and consistently statistically significant associations were found between parents' and children's CVD risk-factors.

Conclusion Parental behaviours like smoking, alcohol consumption, and low physical activity were significantly associated with higher levels of certain CVD risk-factors in children. Strong correlations in CVD risk-factors between family members that are not related to SEP or parental lifestyle suggest a role of genetics in influencing children's CVD risk-factors. Public health policies should target families with unhealthy lifestyles.

\section{P2-139 PERIODONTAL INFLAMMATIONS ARE RELATED TO NITRIC OXIDE LEVELS IN SALIVA}

doi:10.1136/jech.2011.142976i.74

H-D Kim, ${ }^{*}$ D-H Han, H-M Kim, H-S Shin, M-S Kim. Seoul National University, Seoul, Republic of Korea

Introduction Nitric oxide (NO) plays an important role in almost every biological system. The increase in NO in periodontal tissues has been reported in inflammatory periodontal disease, which suggests the production and participation of $\mathrm{NO}$ in the disease process. However, the association between salivary NO levels and periodontitis was rarely reported. The aim of this study was to assess the periodontal health according to the salivary NO levels in Korean elderly.

Methods 203 subjects aged 48-84 years old were cross-sectionally surveyed. All participants underwent oral examination. Age, gender, education level, regular exercise, smoking were evaluated through interview. Periodontal health status was evaluated by clinical attachment loss over $6 \mathrm{~mm}$ (CAL6 $\mathrm{mm}$ ) for 6 points of 12 index teeth (total 72 sites). The number of CAL $6 \mathrm{~mm}$ sites was counted. Unstimulated saliva was collected for $10 \mathrm{~min}$. Salivary NO was determined by the Griess reagent. ANCOVA and Multiple linear regression analyses were applied.

Results After correcting for differences in for age, gender, education, regular exercise, smoking, salivary flow rate, and number of natural teeth, high NO level group showed the highest number of CAL6 $\mathrm{mm}$ sites (15.2 vs 7.0 and 10.8, $\mathrm{p}=0.031$ ). Salivary NO levels had a dose-effect relationship with the number of CAL6 $\mathrm{mm}$ sites $(\beta=0.026, p=0.011)$.

Conclusion NO levels are elevated in individuals with periodontitis. These results reveal that periodontal disease and its severity are related to salivary $\mathrm{NO}$ concentration, indicating that $\mathrm{NO}$ may serve as a potential biological marker for detection and monitoring of periodontitis.

\section{P2-140 DIETARY PATTERNS AND RISK OF METABOLIC SYNDROME AMONG KOREAN POPULATION: THE KOREAN NATIONAL HEALTH AND NUTRITION EXAMINATION SURVEY 2007-2009}

doi:10.1136/jech.2011.142976i.75

${ }^{1} \mathrm{M} \mathrm{K} \mathrm{Kim},{ }^{*}{ }^{2} \mathrm{M}$ Lee, ${ }^{3} \mathrm{C}$ Shin, ${ }^{4} \mathrm{I}$ Baik, ${ }^{1} \mathrm{Y}$ Yun, ${ }^{5} \mathrm{H}$ Okubo, ${ }^{5} \mathrm{~S}$ Sasaki. ${ }^{1}$ Cancer Epidemiology Branch, National Cancer Center, Goyang, Republic of Korea; ${ }^{2}$ Department of Food and Nutrition and Research Institute of Obesity Sciences, Sungshin Women's University, Seoul, Republic of Korea; ${ }^{3}$ Department of Internal Medicine, Korea University Ansan Hospital, Ansan, Republic of Korea; ${ }^{4}$ Department of Foods and Nutrition, College of Natural Sciences, Kookmin University, Seoul, Republic of Korea;
${ }^{5}$ Department of Social and Preventive Epidemiology, Graduate School of Medicine, the University of Tokyo, Tokyo, Japan

An increasing number of studies in Western countries have examined the relationship between dietary pattern, namely the measurement of overall diet by considering the cumulative effects of nutrients, and the risk of metabolic syndrome (MetS). However, such information is absolutely lacking among Asian populations, including Korea, with different subject characteristics and culturespecific dietary habits. This cross-sectional study examined the association between dietary pattern identified by factor analysis and the risk of MetS among Korean population. We used data from the Korean National Health and Nutrition Examination Survey, 2007-2009. The analytical sample included men and women aged 30-80 years with $24 \mathrm{~h}$ recall, anthropometric and clinical measurements $(n=5320)$. MetS was defined based on the Adult Treatment Panel III of the National Cholesterol Education Program criteria as having three or more risk factors using a modified obesity index. We identified three dietary patterns ("Meat \& alcohol", "Unbalanced Korean", and "Diverse") in both sexes. After adjustment for potential confounding factors, subjects in the highest quintile of the "Diverse" dietary pattern had significantly lower risk of MetS compared with those in the lowest in both sexes (multivariate OR: $0.50 ; 95 \% \mathrm{CI}$ 0.27 to 0.90 ; $p$ for trend $=0.174$ for men; multivariate OR: $0.58 ; 95 \%$ CI 0.38 to 0.87 ; p for trend $=0.005$ for women). Other dietary patterns were not associated with the risk of MetS. The result suggests that a diet high in vegetables, fruits, eggs, fish, and meat might reduce the risk of MetS among Korean population.

\section{P2-141 DEFINITION AND VALIDATION OF AN ALGORITHM TO IDENTIFY COPD PATIENTS FROM ADMINISTRATIVE DATABASES}

doi:10.1136/jech.2011.142976i.76

L Bauleo, U Kirchmayer,* V Belleudi, N Agabiti, L Pinnarelli, S Cascini, D Fusco, M Arcà, M Davoli. Department of Epidemiology of the Regional Health Service, Lazio Region, Italy

Introduction Administrative databases are increasingly used to identify patients with chronic conditions, however the optimal methodology for Chronic Obstructive Pulmonary Disease (COPD) is still debated.

Objective To develop and validate an algorithm to identify patients with COPD in Lazio (2625102 residents over 45) linking clinical and administrative data.

Methods From the regional hospitalisation, drug prescription and outpatient registries, through record linkage, we identified patterns of specific drug use (minimum 2 prescription during 12 months) and COPD hospitalisations during a 9-year period in 428 patients with COPD, who attended an outpatient clinic in 2006, and in 2140 people without COPD. Through a Bootstrap-Stepwise procedure we selected COPD associated factors. We validated the algorithm through internal (cross-validation-bootstrap, jack-knife) and external validation (comparison with external COPD patients with confirmed diagnosis).

Results A total of 205611 (7.8\%) COPD patients were identified. Factors associated with COPD were: prescription of $\beta 2$-agonists, anticholinergics, corticosteroids, oxygen, and previous hospitalisation for COPD and respiratory failure. For each patient we estimated an expected probability to suffer from COPD. Depending on the cut-point of expected probability, sensibility (SE) ranged from 0.15 to 0.87 and specificity (SP) from 0.79 to 0.99 . We defined a cutpoint of $0.30(\mathrm{SE}=64 \%$; $\mathrm{SP}=97 \%$ ) to identify the COPD patients. Applying our algorithm on external COPD patients we succeeded to identify $86 \%$ 